\title{
Neutral polyampholyte in an ionic solution
}

\author{
Alexandre Diehl, Marcia C. Barbosa, and Yan Levin \\ Instituto de Física, Universidade Federal do Rio Grande do Sul, Caixa Postal 15051, 91501-970, Porto Alegre, \\ Rio Grande do Sul, Brazil
}

(Received 8 May 1996)

\begin{abstract}
The behavior of a neutral polyampholyte (PA) chain with $N$ monomers, in an ionic solution, is analyzed in the framework of the full Debye-Hückel-Bjerrum-Flory $(\mathrm{DHBjF})$ theory. A PA chain, that in addition to the neutral monomers, also contains an equal number of positively and negatively charged monomers, is dissolved in an ionic solution. For high concentrations of salt and at high temperatures, the PA exists in an extended state. As the temperature is decreased, the electrostatic energy becomes more relevant and at a $T=T_{\theta}$ the system collapses into a dilute globular state, or microelectrolyte. This state contains a concentration of salt higher than the surrounding medium. As the temperature is decreased even further, association between the monomers of the polymer and the ions of the salt becomes relevant and there is a crossover from this globular state to a low temperature extended state. For low densities of salt, the system is collapsed for almost all temperatures and exhibits a first-order phase transition to an extended state at an unphysical low temperature. [S1063-651X(96)00712-X]
\end{abstract}

PACS number(s): 36.20.Ey, 64.60.Cn, 82.35.+t, 87.15.By

\section{INTRODUCTION}

In recent years, there has been a lot of interest in the study of the physical properties of macromolecules. When dissolved in a good solvent, a neutral macromolecule, that is, made of $N$ units (monomers), is characterized by a radius of gyration that scales as $R \sim N^{\nu} a$, with $\nu=3 / 5$. In a bad solvent, as the temperature is lowered, a polymer undergoes a phase transition from an extended state, characterized by $\nu=3 / 5$, to a compact globular state, with $\nu=1 / 3$. At the transition point $T=T_{\theta}$ the system assumes a coil configuration, with $\nu=1 / 2$. The transition resembles the tricritical behavior present in some magnetic materials [1] and can be described by the Flory-de Gennes theory [1-4].

In nature, however, charged polymers are prevalent. They can be divided into two classes, polyelectrolytes and polyampholytes (PAs). The most common example of a polyelectrolyte is a DNA dissolved in water [5]. Upon dilution, the phosphate groups of the DNA molecule lose their $\mathrm{H}^{+}$atoms and become ionized. All the ionized groups of the polyion carry the charge of the same sign. The repulsion between the like charges helps to stretch the molecule and contributes to the overall persistence length of a polyion [6,7].

Proteins, on the other hand, are an example of polyampholytes. A PA molecule can have monomers of either positive or negative charge. Thus two extremes are possible. In one extreme, we can have a neutral PA, in which the number of positive monomers is exactly equal to the number of negative monomers. In the other extreme, all the monomers can be of the same sign, in which case a PA will be equal to a polyelectrolyte. In this paper we shall confine our attention to the case of a neutral PA.

In contrast to a normal polymers, the understanding of PAs is rather poor. This is due to the difficulty of properly accounting for the long-range Coulomb interaction between the monomers of a PA. The long-range electrostatic interaction is qualitatively different from the short-range excluded volume repulsion, that drives the coil-globule transition in a usual uncharged polymer. Consequently, our understanding of the full conformational structure of the PA is rather incomplete.

One of the first attempts to study a neutral PA was done by Edwards, King, and Pincus [8]. They argued, on the basis of the Debye-Hückel (DH) limiting law, that when the polymer chain is in a nonionic solvent, the competition between the net attractive electrostatic energy of the PA and the reduction in its entropy, due to the confinement in a sphere smaller than its natural radius, leads to a transition from an extended coil to a "dilute globular state" (microelectrolyte). The microelectrolyte is characterized by a density smaller than that of the collapsed state of a chain made of neutral monomers. The microelectrolyte picture of a PA was further extended and elaborated in the work of Higgs and Joanny [9], who have concluded that a sufficiently long PA will always be collapsed.

In a recent papers, we have investigated $[10,11]$ the possibility of a different type of coil-globule transition in a neutral nonalternating PA. In the absence of salt, the behavior of a neutral PA, with $N$ charged monomers, was analyzed in the framework of the full DH theory [12], augmented by Bjerrum's idea of ion association [13] and Flory's affine network theory [14] of rubber elasticity. In this approach, allowance was made for the fact that a strong electrostatic attraction between the unlike monomers makes it favorable for them to associate forming intermolecular bridges. The PA molecule then resembles an affine network with the crosslinks of functionality 4 [15]. It was found that at high temperatures the crosslinks are quite rare, and the picture of a PA as existing in a dilute globular state remains unchanged, with the radius of gyration scaling as $R \sim a N^{\nu}$ and $\nu=1 / 3$ (microelectrolyte), as predicted by Edwards et al. [8,9]. As the temperature is decreased, the ionic association becomes energetically favorable. At very low temperatures, the fraction of unassociated charged monomers approaches zero and the PA molecule resembles a microgel, with $\nu=2 / 5$ (compare with the Flory exponent $\nu_{F}=3 / 5$ ). The two states are separated by a first- 
order phase transition. It is interesting to note that unlike the case of an usual neutral polymer, the extended state of PA $(\nu=2 / 5)$ occurs at low temperatures.

In order to analyze the effects of the addition of salt to the polymeric solution [16], Higgs and Joanny [9] extended Edwards, King, and Pincus [8] method, including the electrostatic interaction between the charged monomers of the chain and the free ions of the salt. They considered the case where the density of charges on the PA is of the same order of magnitude as the density of added salt. They have concluded that the only effect of added salt was to renormalize the second virial coefficient, adding an attractive term to it. At high temperatures and in a good solvent, the excluded volume term dominates the attractive $\mathrm{DH}$ contribution and the chain is extended. As the temperature is decreased, the electrostatic contribution balances the excluded volume contribution and the polymer collapses into a dilute globular state.

Higgs and Joanny's theory (HJ), however, is based on the assumption that the density of the polymer is close to the density of salt, which is actually a good approximation only when the densities are quite high. Furthermore, since HJ work in the framework of the linearized DH theory, their conclusions can only be trusted at high temperatures and cannot be extended to the low temperatures, where they find an unphysical divergence of the concentration of ions near the PA.

In order to further explore these issues, we analyze this problem in the framework of the full Debye-Hückel Bjerrum-Flory (DHBjF). The possible association between the counterions and the monomers of the chain is explicitly taken into account. In a good solvent and for density of salt $\rho_{0}>\bar{\rho}(\bar{\rho}$ is the density of polymer in extended state at high temperatures), corresponding to the region where $\mathrm{HJ}$ found the transition, the system has the following behavior. For high temperatures, the repulsion due to the excluded volume dominates over the DH electrostatic attraction and the polymer is extended. The counterion association is insignificant in this region. As the temperature is decreased, the electrostatic energy prevails and at $T=T_{\theta}$ the system collapses into the dilute globular state, in agreement with the HJ predictions. We obtain explicitly the values of the transition temperature for different densities of salt and for different volume fractions of charged monomers. As the temperature is lowered even further, the counterion-monomer association becomes energetically favorable. The number of ions of salt attached to the chain increases. As $T \rightarrow 0$, the fraction of unpaired monomers approaches zero and the PA molecule becomes extended like a usual neutral polymer. Within our approach we find that the instability observed by $\mathrm{HJ}$ is hidden inside the metastable region of the salt solution. Furthermore, beyond the $\mathrm{HJ}$ results, we are able to investigate the properties of a polymer-salt solution when the density of salt is quite small. We find that for $\rho_{0}<\bar{\rho}$, the fluctuation induced attraction between the monomers is strong and the polymer exists in a dilute collapsed state for almost all temperatures. At very low temperatures and low densities, the system undergoes a first-order transition into an extended state.

The paper is organized as follows. In Sec. II our model for PA + salt is presented and the Helmoltz free energy of the composed system is constructed. A full thermodynamic analysis is formulated in Sec. III, in particular, the equations

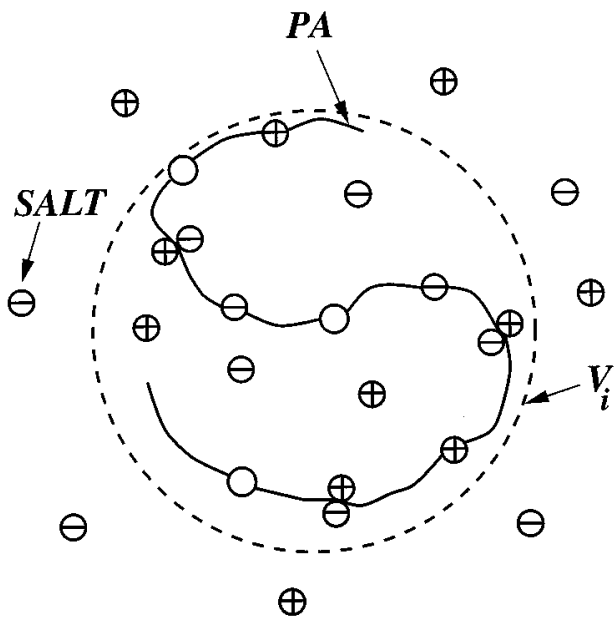

FIG. 1. Schematic drawings of a PA chain with $N$ neutral monomers $\bigcirc,\left(N-N_{N}\right) / 2$ positive monomers $\oplus$, and $\left(N-N_{N}\right) / 2$ monomers $\ominus$, immersed in an ionic solution. The salt ions can associate with the positive and negative monomers of the chain, forming dipolar pairs. The PA is confined to a spherical region of radius $R$.

that determine the equilibrium configurations of the system. In Sec. IV a detailed discussion of our results is presented.

\section{DESCRIPTION OF THE MODEL}

\section{A. Introduction}

We consider a polyampholyte (PA) chain consisting of $N$ constitutional units (hereafter referred to as monomers) of three different types: $N_{N}$ neutral monomers, $\left(N-N_{N}\right) / 2$ positive monomers and $\left(N-N_{N}\right) / 2$ negative monomers, randomly distributed along the chain, each spherical in shape with a diameter $a$. The polymer chain occupies an effective sphere of volume $V_{i}=4 \pi R^{3} / 3$, where $R$, the radius of gyration, measures the end-to-end extension of the polymer. The reduced density of monomers $\rho^{*}=\rho a^{3}$ inside the globule is $\rho^{*}=3 N a^{3} /\left(4 \pi R^{3}\right)$.

The solvent that occupies the total volume $V$ of the system is represented by a uniform medium of a dielectric constant $\epsilon$ in which an amount of simple 1:1 salt has been added. The positive and negative ions of salt dissolved in the solvent have a fixed densities $\rho_{0}=\rho_{0}^{+}+\rho_{0}^{-}=2 \rho_{0}^{+}=2 \rho_{0}^{-}$. The basic approximations of our model are (1) in the limit of large dilution we can neglect the interactions between different PAs and concentrate our attention on one chain. (2) The most relevant interaction between the monomers of the chain and the free ions of salt is of electrostatic nature. For simplicity, the excluded volume interactions between the PA and the salt will be neglected. (3) The short-range interactions between the polymer and nonionic solvent are included in the effective value of the second virial coefficient.

The strong electrostatic interactions make it favorable for the ions of salt to associate with the PA. After the thermal equilibrium is achieved, the chain is composed of three different structures: neutral monomers, free charged monomers, and monomers that have an associated ion of salt attached to them (see Fig. 1). We shall refer to the latter as a "dipolar pair." The total number of monomers can then be written as 


$$
N=N_{N}+N_{p}^{+}+N_{p}^{-}+N_{d}
$$

where $N_{p}^{+}$and $N_{p}^{-}$are the number of unassociated positive and negative monomers and $N_{d}$ is the total number of dipoles. Defining $f$ as the number fraction of charged monomers in the PA, and $x$ as the fraction of charged monomers that form dipoles, we can write

$$
\begin{gathered}
N_{N} \equiv(1-f) N, \text { neutral }, \\
N_{p}^{+} \equiv \frac{f}{2}(1-x) N=N_{p}^{-}, \text {charged } \\
N_{d} \equiv f x N, \text { dipoles }
\end{gathered}
$$

It is then possible to express the densities of neutrals, charges, and dipolar pairs, in terms of the total density of the chain $\rho \equiv N / V_{i}$ by the expressions

$$
\begin{gathered}
\rho_{N} \equiv(1-f) \rho, \\
\rho^{+} \equiv \frac{f}{2}(1-x) \rho=\rho^{-}, \\
\rho_{d} \equiv f x \rho=\rho_{d}^{+}+\rho_{d}^{-},
\end{gathered}
$$

where $\rho_{d}^{+}$and $\rho_{d}^{-}$are the densities of dipoles containing positive and negative monomers, respectively. In principle, ions of salt are different from the monomers of the PA and the dipoles formed of positive and negative monomers have to be distinguished. Since, however, the PA is neutral, in equilibrium $\rho_{d}^{+}=\rho_{d}^{-}$.

The salt particles are free to move along the whole volume of the composed system. In the absence of the PA, the positive and negative charges are uniformly distributed with a constant density $\rho_{0}$. In the presence of PA, due to electrostatic fluctuations, the density of salt near the polymer is not equal to $\rho_{0}$. Therefore, in order to treat the problem in a simple way, we shall divide the total system of volume $V$ into two subregions: "inside" and "outside" the volume $V_{i}$. The density of salt particles outside is uniform and equal to $\rho_{0}=N_{0} / V_{0}$, where $V=V_{i}+V_{0}$. The density of the salt inside the volume $V_{i}$ is $\rho_{s}=\rho_{s}^{+}+\rho_{s}^{-}$. Since there is an equal number of positive and negative free ions, $\rho_{s}^{+}=\rho_{s}^{-}=\rho_{s} / 2$ $\equiv N_{s} / V_{i}$.

\section{B. Helmoltz free energy}

The Helmoltz free energy for the whole system is the sum of two terms $F=F_{i}+F_{0}$. The "inside" free energy $F_{i}$, associated with the subregion where the polymer and the salt coexist, and the "outside" contribution $F_{0}$, for the regions where only salt is present. The free energy in the outside region is composed of (i) an energy due to short-range repulsion; (ii) an electrostatic energy due to the interaction between ions; (iii) and the kinetic-entropic contribution. For the inside region, the Helmoltz free energy $F_{i}$, contains (i) an elastic contribution due to the elongation and contraction of the chain; (ii) an energy due to the monomer-monomer interaction; (iii) an energy due to the ion-ion short range repulsion; (iv) an electrostatic energy of monomer-monomer, salt- salt, and monomer-salt interactions; (v) an entropic energy due to the mixing of the different types of particles present inside the region $V_{i}$.

\section{Elastic free energy}

According to the Flory-de Gennes $[2,14,17]$ theory, the elastic free energy of the chain is [18]

$$
\beta F_{e l}=\frac{3}{2}\left(\alpha^{2}-1\right)-3 \ln \alpha,
$$

where $\alpha=R / R_{0}$ is the expansion factor of the chain, measured relative to the nonstrained Gaussian state with radius $R_{0} \approx a \sqrt{N}$.

\section{Free energy of excluded volume}

Following the usual Flory-de Gennes [1-4] theory, the short-range interactions between the particles of the chain, at low densities, are approximated by a virial expansion

$$
\beta F_{H C}^{p}=\frac{N}{2} W_{1} \rho+\frac{N}{2} W_{2} \rho^{2},
$$

where $W_{1}$ and $W_{2}$ are second and the third virial coefficients.

The excluded volume interaction for the salt is approximated by the free-volume form $[19,20]$

$$
\beta F_{H C}^{s}=-V_{i} \rho_{s} \ln \left(1-B \rho_{s}\right),
$$

where $B$ is chosen to yield appropriate maximal packing densities or to match high- $T$ second virial coefficient. Since we are assuming that the relevant interaction between the monomers and the salt particles is electrostatic, we do not consider the excluded volume repulsion between the free ions inside $V_{i}$ and the monomers. Outside $V_{i}$, the excluded volume contribution to the Helmoltz free energy is

$$
\beta F_{H C}^{0}=-V_{0} \rho_{0} \ln \left(1-B \rho_{0}\right),
$$

and, for small densities $\rho_{0}$, it can be neglected.

\section{Electrostatic term}

The electrostatic interaction between the charges is calculated in the framework of the Debye-Hückel (DH) theory. The standard argument leads to [12,19-21]

$$
\beta F_{\mathrm{DH}}=-\frac{N_{1}}{T^{*}(\kappa a)^{2}}\left[\ln (\kappa a+1)+\frac{(\kappa a)^{2}}{2}-\kappa a\right] .
$$

Inside $V_{i}$, the inverse Debye screening length $\kappa=1 / \xi_{D}$, is determined by all free unassociated charged species, i.e., both monomers and salt ions, so that $(\kappa a)^{2}=4 \pi \rho_{1}^{*} / T^{*}$, where $\rho_{1}^{*}=a^{3} N_{1} / V_{i}=\rho_{s}^{*}+f(1-x) \rho^{*}$ and $T^{*}=k_{B} T \epsilon a / q^{2}$. Outside the volume $V_{i}$, the Debye length is determined by the concentration of salt $(\kappa a)^{2}=\left(\kappa_{0} a\right)^{2}=4 \pi \rho_{0}^{*} / T^{*}$.

The DHBj theory has proven to be successful in giving the correct qualitative picture of a simple electrolyte [1921]. Thus, while inclusion of higher-order contributions, such as dipole-ion and dipole-dipole interactions, improves the quantitative agreement of the theory with experiments [22] and Monte Carlo (MC) simulations [23], most of the important physics is already captured at the $\mathrm{DHBj}$ level [20]. 
As a leading-order approximation we shall, therefore, treat the dipolar clusters as ideal noninteracting specie.

\section{Free energy of mixing}

Inside the volume $V_{i}$, the system is composed of a mixture of neutral, positive and negative monomers, as well as, positive and negative ions of salt, and dipoles made of monomers and associated counterion. If the bonds connecting the monomers were cut, we would have a "gas" made of these species. The ideal gas free energy of the mixture is

$$
\beta F_{i d}=\sum_{j}\left[N_{j} \ln \frac{\rho_{j} \Lambda_{j}^{3 k_{j}}}{\xi_{j}}-N_{j}\right],
$$

where $\Lambda_{j}(T)$ is the mean thermal wavelength, $\Lambda_{j}(T)=h / \sqrt{2 \pi \bar{m}_{j} k_{B} T}, \bar{m}_{j}$ is the geometric mean mass for a cluster of $k_{j}$ units, and $\xi_{j}(T)$ is the internal partition function. The index $j$ specifies all the possible species: positive, negative, and neutral monomers, dipoles, and positive and negative free ions. In our case $k_{N}=k_{-}=k_{+}=k_{s}=1$ and $k_{2}=2$. To simplify the notation, we shall take the masses of both monomers and ions to be equal to $m$; therefore, $\Lambda_{j}(T)=\Lambda$. Since all the monomers are hard spheres, the molecular partition is a constant $\xi_{N}=\xi_{1}=\xi_{s}=\xi=1$. For dipoles, however, the internal partition function is [13]

$$
\xi_{2}\left(T^{*}\right)=4 \pi \int_{a}^{c} e^{a / r T^{*}} r^{2} d r \equiv K\left(T^{*}\right),
$$

where $K\left(T^{*}\right)$ defines an association constant for the formation of bound dipolar pairs. For the cutoff $c$, we use the Bjerrum's choice $a / 2 T^{*}$ which corresponds to the inflection point of the integral as a function of $c$. The Bjerrum equilibrium constant is

$$
\begin{aligned}
K\left(T^{*}\right)= & \frac{2 \pi a^{3}}{3\left(T^{*}\right)^{3}}\left[\operatorname{Ei}\left(\frac{1}{T^{*}}\right)-\operatorname{Ei}(2)+e^{2}\right]-\frac{2 \pi a^{3}}{3} e^{1 / T^{*}} \\
& \times\left[2+\frac{1}{T^{*}}+\frac{1}{\left(T^{*}\right)^{2}}\right] .
\end{aligned}
$$

As was shown by Ebeling [24] the low temperature asymptotic value of this constant is in exact agreement with the cluster expansion [25].

While the ions of the salt are free to move independently of each other, the monomers of PA are constrained by the bonds that maintain the integrity of the chain. Equation (9), therefore, overestimate the entropic free energy by the amount equivalent to the free energy of the ideal gas composed of $N$ particles. The free energy of mixing is therefore,

$$
\begin{aligned}
\beta F_{M I X}= & \sum_{j}\left[N_{j} \ln \frac{\rho_{j} \Lambda_{j}^{3 k_{j}}}{\xi_{j}}-N_{j}\right]-\left[N \ln \frac{\rho \Lambda^{3}}{\xi}-N\right] \\
= & V_{i} \rho_{N} \ln \Lambda^{3} \rho_{N}+V_{i} \rho^{+} \ln \Lambda^{3} \rho^{+}+V_{i} \rho^{-} \ln \Lambda^{3} \rho^{-} \\
& +V_{i} \rho_{d}^{+} \ln \Lambda^{6} \frac{\rho_{d}^{+}}{K\left(T^{*}\right)}+V_{i} \rho_{d}^{-} \ln \Lambda^{6} \frac{\rho_{d}^{-}}{K\left(T^{*}\right)} \\
& +V_{i} \rho_{s}^{+} \ln \Lambda^{3} \rho_{s}^{+}+V_{i} \rho_{s}^{-} \ln \Lambda^{3} \rho_{s}^{-}-V_{i} \rho \ln \Lambda^{3} \rho-V_{i} \rho_{s}^{+} \\
& -V_{i} \rho_{s}^{-} .
\end{aligned}
$$

Outside, in $V_{0}$, the entropic contribution to the free energy is given by an ideal gas free energy

$$
\beta F_{i d}^{0}=V_{0} \rho_{0}^{+} \ln \Lambda^{3} \rho_{0}^{+}+V_{0} \rho_{0}^{-} \ln \Lambda^{3} \rho_{0}^{-}-V_{0} \rho_{0}^{+}-V_{0} \rho_{0}^{-} .
$$

\section{THERMODYNAMICS OF THE SYSTEM}

The equilibrium configuration of the PA + salt system is determined by the minimization of the Gibbs free energy. Since the total system is composed of two subregions, the total Gibbs free energy can be written as $G \equiv G_{i}+G_{0}$, where $G_{i}$ is the Gibbs free energy of the subsystem formed by the "inside" region and $G_{0}$ is the Gibbs free energy for "outside" region, $V_{0}$. The minimization of the total free energy implies that $\delta G \equiv \delta G_{i}+\delta G_{0}=0$ and, consequently,

$$
\begin{aligned}
0= & \frac{\partial G_{i}}{\partial V_{i}} d V_{i}+\frac{\partial G_{0}}{\partial V_{0}} d V_{0}+\frac{\partial G_{i}}{\partial N_{p}^{+}} d N_{p}^{+}+\frac{\partial G_{i}}{\partial N_{p}^{-}} d N_{p}^{-} \\
& +\frac{\partial G_{i}}{\partial N_{d}^{+}} d N_{d}^{+}+\frac{\partial G_{i}}{\partial N_{d}^{-}} d N_{d}^{-}+\frac{\partial G_{i}}{\partial N_{s}^{+}} d N_{s}^{+}+\frac{\partial G_{i}}{\partial N_{s}^{-}} d N_{s}^{-} \\
& +\frac{\partial G_{0}}{\partial N_{0}^{+}} d N_{0}^{+}+\frac{\partial G_{0}}{\partial N_{0}^{-}} d N_{0}^{-}
\end{aligned}
$$

The total volume of the system is fixed, $V=V_{i}+V_{0}$, which leads to the condition $\delta V_{i}=-\delta V_{0}$ and, consequently, to the equality of the osmotic pressures $P \equiv-\partial G / \partial V$ inside and outside the globule,

$$
P_{i} \equiv P_{p}+P_{s}=P_{0}
$$

In the above expression, our first condition for equilibrium, $P_{p}, P_{s}$, and $P_{0}$ are the osmotic pressures of the polymer, salt inside the volume $V_{i}$, and salt in $V_{0}$, respectively.

Now, since the total number of ions of salt, $N_{s}^{-}+N_{s}^{+}+N_{d}^{+}+N_{d}^{-}+N_{0}^{-}+N_{0}^{+}$, and the total number of monomers that contain charges, $N_{p}^{-}+N_{p}^{+}+N_{d}^{-}+N_{d}^{+}$(inside $V_{i}$ ), are constants, we have $\delta N_{p}=-\delta N_{d}$ (where $N_{d}=N_{d}^{+}+N_{d}^{-}$and $N_{p}=N_{p}^{-}+N_{p}^{+}$) and $\delta N_{0}=-\delta N_{d}-\delta N_{s}$ (where $N_{s}=N_{s}^{-}+N_{s}^{+}$and $N_{0}=N_{0}^{-}+N_{0}^{+}$). Furthermore, since we are considering a neutral PA and a neutral salt solution, the chemical potentials, $\mu_{s}^{ \pm} \equiv \partial G / \partial N_{s}^{ \pm}$and $\mu_{p}^{ \pm} \equiv \partial G / \partial N_{p}^{ \pm}$for negative and positive species are equal $\mu_{s}^{-}=\mu_{s}^{+}=\mu_{s}$ and $\mu_{p}^{-}=\mu_{p}^{+}=\mu_{p}$. Therefore, Eq. (14) becomes

$$
0=-\mu_{p} d N_{d}+\mu_{d} d N_{d}+\mu_{s} d N_{s}-\mu_{0}\left(d N_{s}+d N_{d}\right)=0,
$$

where $\mu_{p}$ and $\mu_{d}$ are the chemical potentials of the charged monomers and the dipoles along the chain and $\mu_{s}$ and $\mu_{0}$ are the chemical potentials of free salt inside and outside the volume $V_{i}$, respectively. Since the number of dipole pairs and free ions are independent variables, the Eq. (16) leads to two additional equilibrium conditions: the chemical association

$$
\mu_{p}+\mu_{s}=\mu_{d}
$$


and the condition for free exchange of salt between the "inside" and the "outside" regions

$$
\mu_{0}=\mu_{s} .
$$

Instead of working with a full system composed of both the "inside"' and the "outside" regions, we can confine our attention strictly to the "inside" region, at the expanse of introducing some Lagrange multipliers. This leads to an effective Gibbs free energy

$$
\beta \mathcal{G}=\beta F+\beta P_{0} V-\beta \mu_{0} N_{s}-\beta \mu_{0} N_{d},
$$

where $F$ is the Helmoltz free energy of the "inside" region,

$$
\beta F=\beta F_{e l}+\beta F_{H C}^{p}+\beta F_{H C}^{s}+\beta F_{D H}+\beta F_{M I X},
$$

and the pressure $P_{0}$ and the chemical potential $\mu_{0}$ play the role of Lagrange multipliers. All the thermodynamic information about the "inside" region is now contained in $\mathcal{G}$. The equilibrium state of the $\mathrm{PA}+$ salt subsystem is determined by the minimization of $\mathcal{G}, \delta \mathcal{G}=0$,

$$
\begin{aligned}
\left(\frac{\partial \beta F}{\partial V}\right. & \left.+\beta P_{0}\right) d V+\left(\frac{\partial \beta F}{\partial N_{s}}-\beta \mu_{0}\right) d N_{s}+\left(\frac{\partial \beta F}{\partial N_{d}}-\beta \mu_{0}\right) d N_{d} \\
& +\frac{\partial \beta F}{\partial N_{p}} d N_{p}=0
\end{aligned}
$$

Making once again use of the conservation of the number of charged monomers and of the number of particles of salt, it is not hard to show that this reduces to three equilibrium Eqs. (15), (17), and (18), respectively.

To find the quilibrium state of the PA + salt system, these equations must be solved simultaneously. Explicitly, the conditions for equality of pressures and for free exchange of salt between the "inside" and the "outside" regions are, respectively,

$$
\begin{aligned}
\rho_{s}+ & \frac{B \rho_{s}^{2}}{1-B \rho_{s}}+\frac{1}{8 \pi a^{3}}\left[2 \ln (z+1)-\frac{z(z+2)}{z+1}\right]-\frac{3\left(\alpha^{2}-1\right)}{4 \pi a^{3} \alpha^{3} N^{3 / 2}} \\
& +\frac{W_{1}}{2} \rho^{2} \\
= & \rho_{0}+\frac{B \rho_{0}^{2}}{1-B \rho_{0}}+\frac{1}{8 \pi a^{3}}\left[2 \ln \left(z_{0}+1\right)-\frac{z_{0}\left(z_{0}+2\right)}{z_{0}+1}\right],
\end{aligned}
$$

where $z \equiv \kappa a=\sqrt{4 \pi \rho_{1}^{*} / T^{*}}$ and $z_{0} \equiv \kappa_{0} a=\sqrt{4 \pi \rho_{0}^{*} / T^{*}}$ and,

$$
\begin{aligned}
\frac{\left(1-B \rho_{s}\right) \rho_{0}}{\left(1-B \rho_{0}\right) \rho_{s}}= & \exp \left[-\frac{1}{2 T^{*}}\left[\frac{z}{z+1}-\frac{z_{0}}{z_{0}+1}\right]\right. \\
& \left.+\frac{B \rho_{s}}{1-B \rho_{s}}-\frac{B \rho_{0}}{1-B \rho_{0}}\right] .
\end{aligned}
$$

The condition of chemical equilibrium between the dipoles and the monopoles reduces to,

$$
\frac{2 x}{(1-x) \rho_{s}}=\frac{K\left(T^{*}\right)}{1-B \rho_{s}} \exp \left[-\frac{1}{T^{*}} \frac{z}{z+1}+\frac{B \rho_{s}}{1-B \rho_{s}}\right] \text {. }
$$
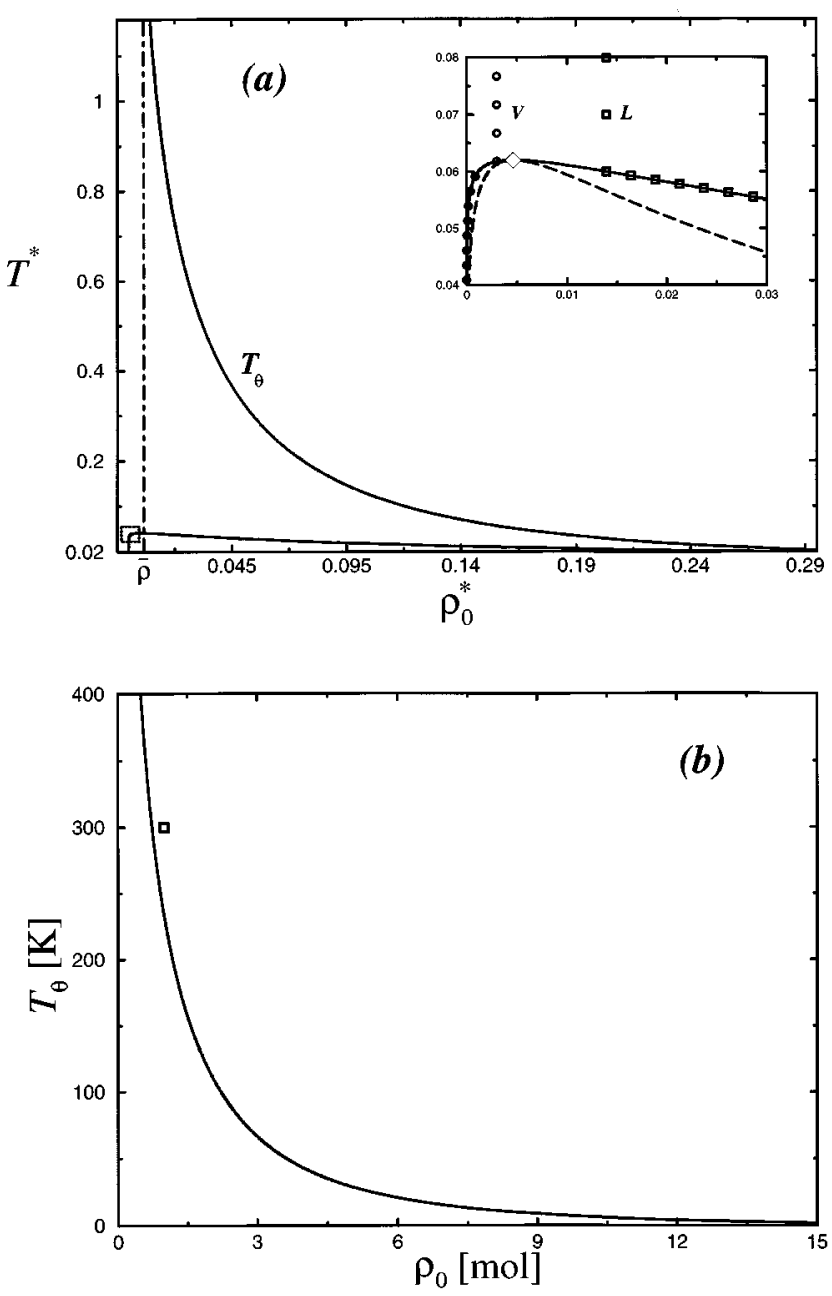

FIG. 2. (a) The coexistence curve for the simple 1:1 salt, for the restricted primitive model (RPM) according to Debye-Hückel (DH) theory, with hard-core repulsive contributions. The region inside the dotted box is represented in the inset. The critical point (open diamond) is $T_{c}^{*} \approx 0.06204$ and $\rho_{c}^{*} \approx 0.00467$. The two possible paths for the density of salt $\rho_{0}$ are represented by $(V)$ on the vapor side of coexistence curve (open circles) and $(L)$ on the liquid side of coexistence curve (open squares). The dashed line represents the HJ line of instability, and the dotted-dashed line is the minimum density $\bar{\rho}$ of the polymer. The solid line represents the $\theta$ temperature $T_{\theta}$; (b) The transition temperature $T_{\theta}$ in $K$ as a function of density in moles (mol). In Eq. (B5) we have used the hydrated diameter of counterions $a=4 \AA$ and the hard sphere second virial term $W_{1}$ $=4 \pi a^{3} / 3$. The square represents the only experimentally available point from Ref. [26].

The equilibrium configuration of the PA, for a fixed $T$ and $\rho_{0}$, is given by the solutions of the coupled Eqs. (22), (23), and (24).

\section{RESULTS AND DISCUSSION}

For a fixed temperature and the external density $\rho_{0}$, these equations can now be solved to yield the values of $\alpha, x$, and $\rho_{s}$. However, as the temperature is lowered, below $T_{c}^{*}$ (see Appendix A for details), the salt solution phase separates into a high (liquid) and low (vapor) density phases. A PA then finds itself either in the high or low density phase of the 


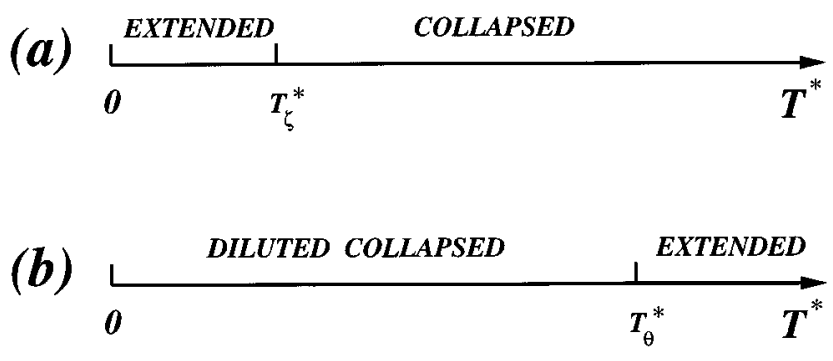

FIG. 3. Schematic phase diagram for: (a) $\rho_{0}$ on the vapor side and (b) $\rho_{0}$ on the liquid side of the coexistence curve. The temperature $T_{\zeta}^{*}$ is very low.

salt solution (see Fig. 2). We shall assume that the PA will be in the high density phase if the original density $\rho_{0}>\rho_{c}$, where $\rho_{c}$ is the critical point density for the pure salt solution $[19,20]$, and in the low density phase $\rho_{0}<\rho_{c}$. We chose a path such that, as the temperature is lowered, the density of the salt remains constant and equal to $\rho_{0}$ up to $T_{\sigma}^{*}$ [the temperature at which $\rho_{0}=\rho_{\sigma}\left(T_{\sigma}^{*}\right)$, where $\rho_{\sigma}$ is the coexistence curve boundary]. Below $T_{\sigma}^{*} \rho_{0}=\rho_{\sigma}\left(T^{*}\right)$.

In order to obtain the phase diagram of the system, we assumed $W_{1}=4 \pi a^{3} / 3$, the value corresponding to the second virial coefficient for the gas of hard spheres, $N=100$ and $f=1$. We chose two different paths: one on the vapor side of the coexistence curve, represented by the path $V$ in Fig. 2 and another on the liquid side of the coexistence curve, represented by the path $L$.

First, the high temperature density of the salt outside the volume $V_{i}$ was fixed at $\rho_{0}^{*}=0.0139>\bar{\rho}^{*}$ (corresponding to $T_{\sigma}^{*}=0.06$ ), which implies a quench on the liquid side of the coexistence curve as shown in Fig. 2 . Here, $\vec{\rho}^{*} \simeq 0.0065$ is the reduced density of the polymer in the extended state, associated with $\alpha=\bar{\alpha}$, where $\alpha$ is the solution of Eq. (27), with $W_{1}>0$ [see Fig. 4(b)]. Solving Eqs. (22), (23), and (24), the following phase diagram arises (see Fig. 3 and Fig. 4). At high temperatures, the excluded volume repulsion is the dominant interaction and the polymer is extended (scaling as $\nu=3 / 5$ ). As the temperature is decreased, the fluctuation induced electrostatic attraction increases and, at a temperature $T_{\theta}^{*}$ the polymer collapses into a dilute globular state, the microelectrolyte (see Appendix B for details). As the temperature is decreased even further, association between the monomer and the counterions begins to takes place. The values of $x$ and $\alpha$ smoothly increases and the polymer expands. Meanwhile, the density of the salt inside the globule approaches from above the value of the density outside the volume $V_{i}, \rho_{\sigma}^{l}(T)$. In the Appendix B this collapse transition is analyzed in detail and the transition temperature $T_{\theta}^{*}$ is calculated in the framework of the full DH theory for different values of salt density and fraction of charged monomers. The unphysical divergence of the density of salt in the vicinity of a PA, encountered by $\mathrm{HJ}$ is no longer present, being hidden inside the coexistence region, as shown in Fig. 2.

Next, the high temperature density of salt outside $V_{i}$ was fixed at $\rho_{0}^{*}=0.003<\rho_{c}^{*}\left(\right.$ at $\left.T_{\sigma}^{*}=0.0618\right)$, which implies a path on the vapor side of the coexistence curve. The solution of Eqs. (22), (23), and (24) leads to the phase diagram for this region, shown in Fig. 3 and Fig. 5. Even for high temperatures, the electrostatic energy is quite large and, conse-
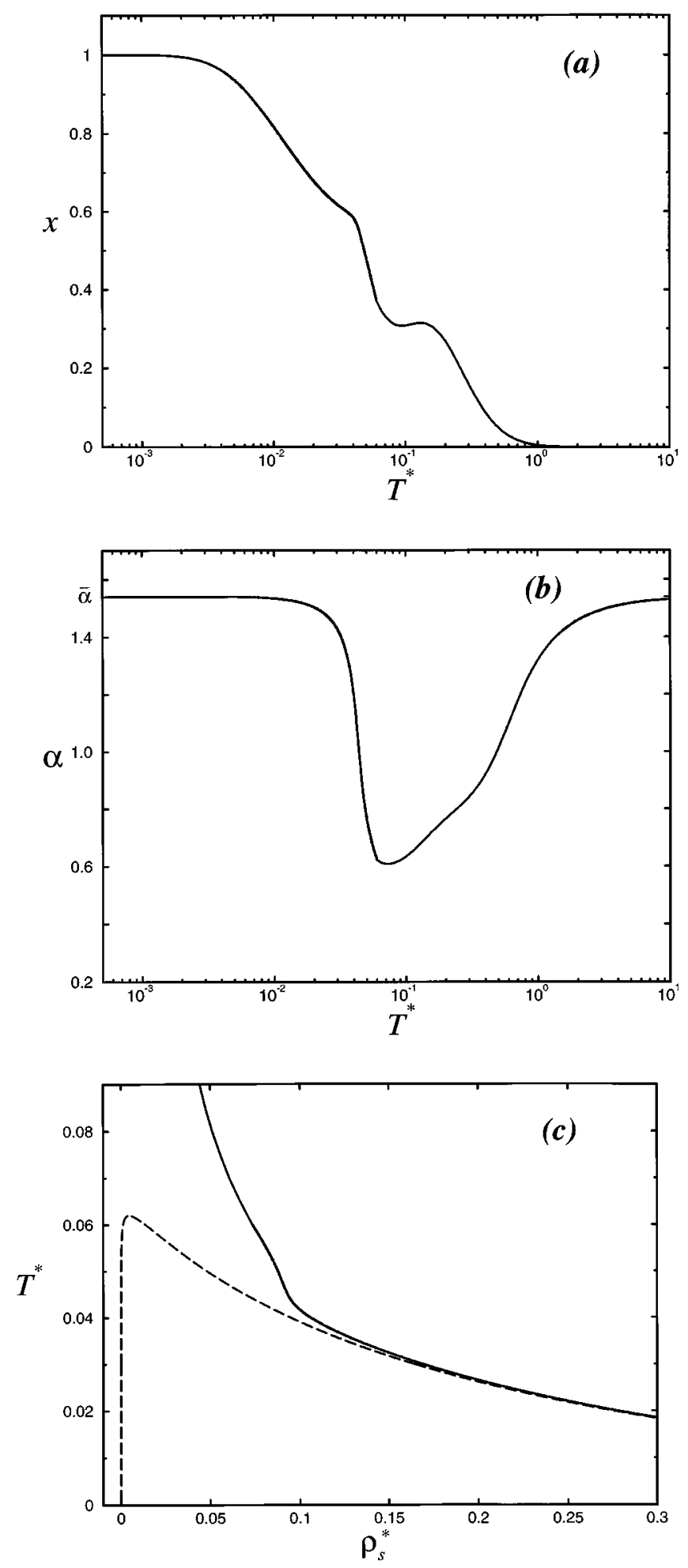

FIG. 4. (a) Fraction of dipoles $x$, (b) expansion factor $\alpha$, and (c) reduced density of salt inside of spherical region $\rho_{s}^{*}$ for a quench on the liquid side of the coexistence curve, is shown against the temperature, for $N=100, f=1$ and $W_{1}=4 \pi a^{3} / 3$. The dashed line in (c) represents the coexistence boundary.

quently, the polymer is found in the dilute globular state for any physical temperature. Therefore, no high temperature collapse transition is found, when the density of salt is $\rho_{0}<\overline{\boldsymbol{\rho}}$. As the temperature is lowered, however, the ionic association becomes energetically favorable and the free ions 

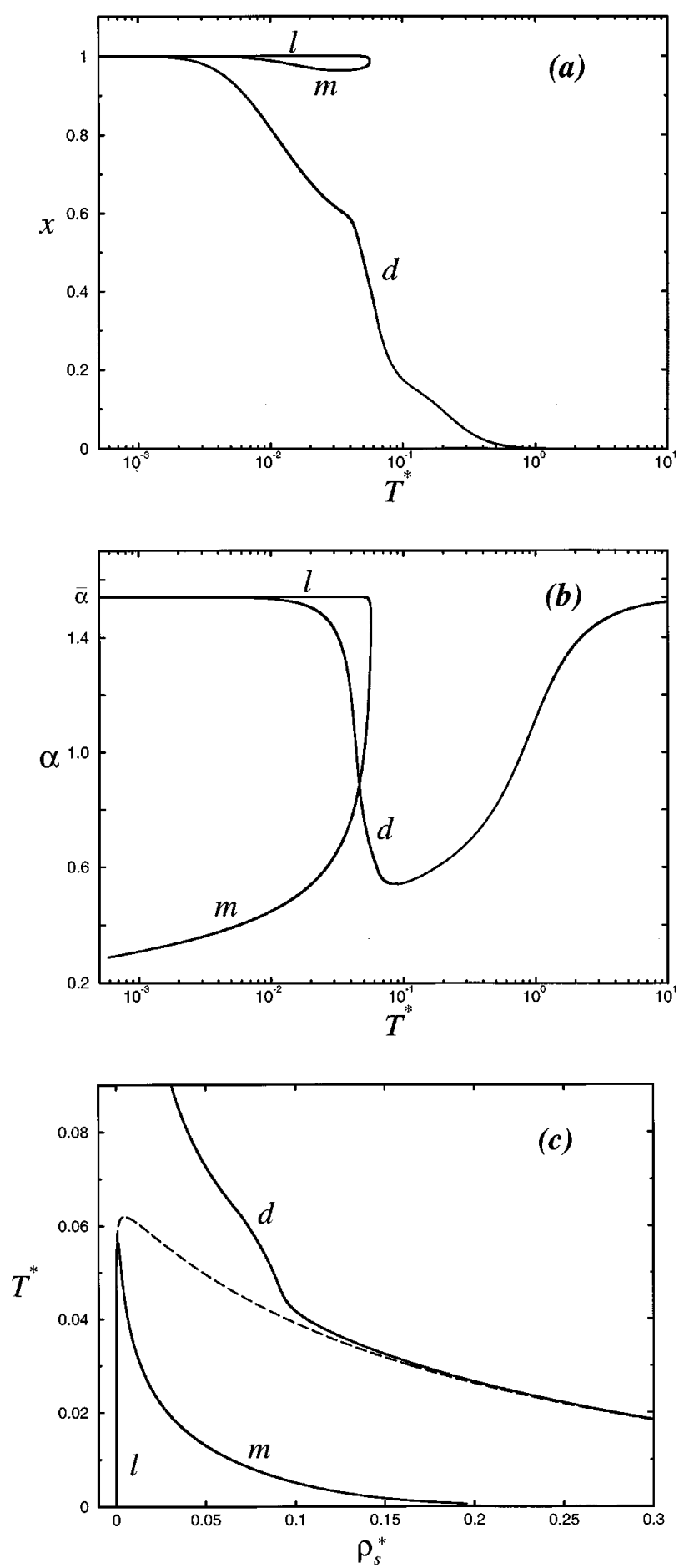

FIG. 5. (a) Fraction of dipoles $x$, (b) expansion factor $\alpha$, and (c) reduced density of salt inside of spherical region, for a quench on the vapor side of the coexistence curve, is shown against the temperature, for $N=100, f=1$ and $W_{1}=4 \pi a^{3} / 3$. The stable branch $d$ represents a dilute collapsed state, $l$ represents a stable extended state and $m$ is a metastable state. Notice how the density of salt inside the globule asymptotes to the liquid side of the phase boundary in $d$, even if the density outside $\rho_{0}$ is fixed at the vapor phase.

of the salt to condense onto the charged monomers of the PA. Indeed, in this region $x, \rho_{s}$, and $\alpha$ are not a single valued function. Besides the dilute globular state (designed with $d$ in Fig. 5), there are two other branches: one meta- stable (designed with $m$ in Fig. 5) and another stable branch (assigned by $l$ in Fig. 5). In the presence of a good solvent, the polymer in this last stable branch is actually extended. At $T^{*} \approx T_{\zeta}^{*}$ (see Fig. 3), there is a first-order phase transition between the dilute globular state and the low temperature extended state. This transition actually happens at such a low temperature (see Appendix $\mathrm{C}$ for details), that it is even difficult to give a good estimate numerically.

For a quench on vapor side, as the temperature is decreased, the density of the salt inside $V_{i}, \rho_{s}$, exhibits an interesting behavior. Even if the density outside $V_{i}$ is fixed at the vapor phase, the density inside $V_{i}$ is at $\rho_{\sigma}^{l}\left(T^{*}\right)$ [see Fig. $5(\mathrm{c})]$. The salt actually separates in two phases, a liquid phase inside the globule and a vapor phase outside. Therefore, even for a very low density of the salt outside the globule $\rho_{0}=\rho_{\sigma}^{v}\left(T^{*}\right)$ the density of salt inside is quite large $\left[\rho_{s}=\rho_{\sigma}^{l}\left(T^{*}\right)\right]$.

Finally, the density of the salt was fixed at $\rho_{c}^{*}<\rho_{0}^{*}$ $=0.00636<\bar{\rho}^{*}\left(\right.$ at $\left.T^{*}=0.0619\right)$, which implies a quench on the liquid side of the coexistence curve, but in the region where the collapse transition is absent. In this case, like in the preceding situation, the polymer is in the dilute globular state for almost all temperatures. Differently from what has happened on the vapor side of the coexistence curve, at low temperatures there is a smooth crossover from the globular state to the extended state.

As we pointed out in the last two cases, when the density of the salt is small $\rho_{0}<\bar{\rho}$ the DH contribution is governed by the density $\rho$ of polymer. For large temperatures, there are almost no dipoles, $x \rightarrow 0$ and the density of salt inside the volume $V_{i}$ approaches the value outside $\rho_{0}$. In this region $z$ becomes small and the DH limiting law can be used in Eq. (22), which becomes

$$
-\frac{3\left(\alpha^{2}-1\right)}{4 \pi a^{3} \alpha^{3} N^{3 / 2}}+\frac{W_{1}}{2} \rho^{2}+W_{E} \rho^{3 / 2}=0,
$$

where $W_{E} \equiv f a^{3 / 2} \sqrt{4 f \pi} / 6\left(T^{*}\right)^{3 / 2}$. In the $N \rightarrow \infty$ limit, the solution of Eq. (25) implies a finite value for the density $\rho$ and, consequently, the size of the polymer scales as

$$
R=a_{0} N^{1 / 3},
$$

where $a_{0} \equiv 3 T^{*}\left(W_{1}^{*} / 4 \pi\right)^{2 / 3} a / f(1-x)$, with $W_{1}^{*} \equiv W_{1} / a^{3}$ as a dimensionless parameter, corresponding to the quality of the solvent.

Below $T_{\zeta}^{*}$ the conformation of the PA is governed by branch $l$ (see Appendix C). Since along this branch all the monomers are neutralized by the counterion, the attractive electrostatic interaction is absent and in Eq. (22) the hardcore repulsive term (repulsive) has to be balanced by the elastic term (attractive). In this case, we find that

$$
-\frac{3\left(\alpha^{2}-1\right)}{4 \pi a^{3} \alpha^{3} N^{3 / 2}}+\frac{W_{1}}{2} \rho^{2}+W_{2} \rho^{3}=0,
$$

which is the same as for a neutral polymer:

(i) If $W_{1} \gg 0$ (good solvent), the elastic term $-3\left(\alpha^{2}-1\right) / 4 \pi a^{3} \alpha^{3} N^{3 / 2}$ balances the hard-core contribution 
$W_{1} \rho^{2} / 2$ and gives a radius of Gyration $R=a_{1} N^{3 / 5}$, where the amplitude $a_{1} \equiv\left(3 W_{1}^{*} / 8 \pi\right)^{1 / 5} a$. So, the PA is in an extended state;

(ii) if $W_{1}=0$ ( $\theta$ solvent), the second virial term is zero, and the elastic term balances the third virial $W_{2} \rho^{3}$. In this case the radius scales as $R=a_{2} N^{1 / 2}$, where $a_{2}$ $\equiv\left[9 W_{2}^{*} /(4 \pi)^{2}\right]^{1 / 8} a$, and $W_{2}^{*} \equiv W_{2} / a^{6}$. In this case, the PA behaves like a coil;

(iii) if $W_{1} \ll 0$ (poor solvent), the second virial coefficient $\left|W_{1}\right| \rho^{2} / 2$ balances the third virial $W_{2} \rho^{3}$ and the radius of Gyration scales as $R=a_{3} N^{1 / 3}$, where $a_{3}$ $\equiv\left(3 W_{2}^{*} / 2 \pi\left|W_{1}^{*}\right|\right)^{1 / 3} a$. Thus, the PA is in a dense collapsed state.

Unfortunately this interesting behavior will be impossible to observe experimentally since $T_{\zeta}^{*}$ is too low.

\section{CONCLUSION}

In this work, we have analyzed the behavior of a neutral polyampholyte in the presence of salt, using the full DebyeHückel-Bjerrum-Flory (DHBjF) theory. Allowing for the association between the charged monomers and the free ions of salt, we found that when the density of salt is large $\rho_{0}>\bar{\rho}$, at high temperatures the PA is extended. As the temperature is decreased, there is a collapsed transition to a dilute collapsed state at $T=T_{\theta}$. As the temperature is decreased even further, the free ions of the salt associate with the charged monomers, decreasing the electrostatic attraction. In fact, at zero temperature, all of the monomers are neutralized and, since there is no free charges left, the chain behaves as a usual neutral polymer. Therefore, in the presence of a good solvent, there is a crossover from a dilute globular state to an extended state at low temperatures. If the density of salt is small $\rho_{0}<\bar{\rho}$ the polymer is in the dilute collapsed state for almost all temperatures.

This behavior is similar to that found experimentally by Corpart and Candau [26] in their study of an aqueous solution of neutral PAs. They observed that a neutral chain containing equal number of positive and negative monomers is insoluble in a low density electrolyte. This is in agreement with our conclusion that the PA will remain collapsed if the density of salt is less than $\bar{\rho}$. If the density of salt is increased above $\bar{\rho}$, for $T>T_{\theta}$, the chain swells and becomes soluble. This is indeed what was observed in the experiments of [26].

Our analysis extends the work of HJ. We obtain explicitly the value of the transition temperature $T_{\theta}$ (Fig. 2) as a function of salt density. Furthermore, we demonstrate that the unphysical instability observed by $\mathrm{HJ}$ is a result of their use of the Debye-Hückel limiting law and is hidden inside the metastable region when the full DHBj theory is applied.

\section{ACKNOWLEDGMENTS}

This work was supported in part by $\mathrm{CNPq}-$ Conselho Nacional de Desenvolvimento Científico e Tecnológico and FINEP Financiadora de Estudos e Projetos, Brazil.

\section{APPENDIX A: COEXISTENCE CURVE FOR THE SALT}

The salt solution exhibits two possible phases, liquid (high density), and vapor (low density). The coexistence curve boundary $\rho_{\sigma}\left(T^{*}\right)$ between these phases is given by the solutions of the coupled equations

$$
P\left(\rho_{\sigma}^{v}\right)=P\left(\rho_{\sigma}^{l}\right),
$$

and

$$
\mu\left(\rho_{\sigma}^{v}\right)=\mu\left(\rho_{\sigma}^{l}\right),
$$

where $P \equiv-\partial \beta F / \partial V$ and $\mu \equiv \partial \beta F / \partial N$. The Helmoltz free energy $\beta F=\beta F_{H C}+\beta F_{D H}+\beta F_{i d}$ is given by the Eqs. (7), (8), and (13). Explicitly,

$$
P\left(\rho_{\sigma}\right)=\rho_{\sigma}+\frac{B \rho_{\sigma}^{2}}{1-B \rho_{\sigma}}+\frac{1}{8 \pi a^{3}}\left[2 \ln (z+1)-\frac{z(z+2)}{z+1}\right]
$$

and

$$
\mu\left(\rho_{\sigma}\right)=-\frac{1}{2 T^{*}} \frac{z}{z+1}+\ln \Lambda^{3} \frac{\rho_{\sigma}}{2}-\ln \left(1-B \rho_{\sigma}\right)+\frac{B \rho_{\sigma}}{1-B \rho_{\sigma}},
$$

where $z=\sqrt{4 \pi \rho_{\sigma}^{*} / T^{*}}$. The resulting coexistence curve, for $B=4 a^{3} / 3 \sqrt{3}$ (bcc packing), is shown in Fig. 2 and, for low temperatures, the vapor and liquid branches are given analytically by

$$
\begin{aligned}
\rho_{\sigma}^{v} \approx & \frac{1}{a^{3}} \exp \left[-\frac{1}{2 T^{*}}+\frac{1}{2}\left(\frac{1}{\pi \rho_{M}^{*} T^{*}}\right)^{1 / 2}\right. \\
& \left.+\left(-\frac{1}{2}+\frac{1}{8 \pi \rho_{M}^{*}}\right) \ln T^{*}-\ln 4 \sqrt{\frac{\pi}{\rho_{M}^{*}}}-\frac{\ln 4 \pi \rho_{M}^{*}}{8 \pi \rho_{M}^{*}}\right]
\end{aligned}
$$

and

$$
\begin{aligned}
\rho_{\sigma}^{l} \approx & \frac{1}{a^{3}} \rho_{M}^{*}\left[1-4 \sqrt{\pi \rho_{M}^{*} T^{*}}-2 T^{*} \ln \frac{1}{T^{*}}\right. \\
& \left.-\left(2 \ln 4 \pi \rho_{M}^{*}-2-8 \pi \rho_{M}^{*}\right) T^{*}\right],
\end{aligned}
$$

where $\rho_{M}^{*} \equiv a^{3} / B$ is the maximum packing density. As the temperature $T^{*} \rightarrow T_{c}^{*} \quad \rho_{\sigma}^{l}-\rho_{\sigma}^{v} \rightarrow 0$ and at the critical point $\rho_{\sigma}^{v}=\rho_{\sigma}^{l}=\rho_{c}$. The critical temperature and density are $T_{c}^{*} \approx 0.06204$ and $\rho_{c}^{*} \approx 0.00467$. In this case, the reduced temperature is only $9 \%$ higher than the $\mathrm{MC}$ estimate $\left(T_{c}^{*}=0.057 \pm 1_{5}, \rho_{c}^{*}=0.030 \mp 8\right)[23]$, but the critical density is too small. The low value of critical density is due to the fact that $\mathrm{DH}$ theory omits important nonlinear configurations, such as formation of cluster, dipoles, quadrupoles, etc. This configurations can be reintroduced into the theory by allowing for the existence of equilibrium between monopoles and clusters. At the simplest level that we are considering in this paper, salt dipoles are taken as neutral species that do not interact electrostatically with PA, and can be ignored.

\section{APPENDIX B: THE TEMPERATURE $T_{\theta}^{*}$}

In this Appendix we study the collapsed transition present at high temperatures and high concentration of salt. We also compare our results with those of Higgs and Joanny [9]. At 
high temperatures, the density of salt $\rho_{s}$ inside the volume $V_{i}$ is slightly higher than $\rho_{0}$ (see Fig. 4) and, consequently, we can write

$$
\rho_{s}=(1+\delta) \rho_{0},
$$

where $\delta \ll 1$.

The density of free charges along the polymer can always be defined as $\rho=\gamma \rho_{0}$. In this case, the total density of charges inside $V_{i}$ is

$$
\rho_{1}=\rho_{0}(1+\delta+\widetilde{\gamma})
$$

where $\tilde{\gamma}=f \gamma$.

If $\rho_{0}<\bar{\rho}, \tilde{\gamma}>1$ and, therefore, at high temperatures, where association becomes irrelevant, the electrostatic contribution to the free energy scales as $\rho^{3 / 2}$. This attractive interaction is balanced by second virial term $W_{1} \rho^{2} / 2$, which forces PA to collapse.

If the density of salt is bigger than a fixed value $\rho_{0}>\bar{\rho}$ we have $\widetilde{\gamma}<1$. Therefore, in this case, $\delta$ and $\widetilde{\gamma}$ can be treated as small parameters. Solving Eq. (23) for $\delta$ up to second order in $\tilde{\gamma}$ gives

$$
\begin{aligned}
\delta= & \tilde{\gamma} \frac{1}{4 T^{*}} \frac{z_{0}}{\left(z_{0}+1\right)^{2}}\left[\frac{1}{\left(1-B \rho_{0}\right)^{2}}-\frac{1}{4 T^{*}} \frac{z_{0}}{\left(z_{0}+1\right)^{2}}\right]^{-1} \\
& -\widetilde{\gamma}^{2} \frac{1}{16 T^{*}} \frac{z_{0}}{\left(z_{0}+1\right)^{4}\left(1-B \rho_{0}\right)^{3}} \\
& \times\left[\frac{1}{\left(1-B \rho_{0}\right)^{2}}-\frac{1}{4 T^{*}} \frac{z_{0}}{\left(z_{0}+1\right)^{2}}\right]^{3}\left[\frac{\left(3 z_{0}+1\right)\left(z_{0}+1\right)}{1-B \rho_{0}}\right. \\
& \left.-\frac{1}{2 T^{*}} z_{0}\left(1-3 B \rho_{0}\right)\right] .
\end{aligned}
$$

Assuming that both the monomers and the ions of salt are point particles $(B=0)$ and expanding in $z_{0}$, to leading-order the expression above reduces to Eq. (3.7) of Higgs and Joanny's paper [9]. Unlike their analysis, however, we find that when the full DH theory is used, the unphysical divergence at $1 /\left(1-B \rho_{0}\right)^{2}=z_{0} / 4 T^{*}\left(z_{0}+1\right)^{2}$ is actually located inside the coexistence curve. Since the density of salt is always outside the coexistence region, this divergence is never encountered.

The scaling form of the radius of gyration is then given by substituting Eq. (B3) into Eq. (22)

$$
\begin{aligned}
& -\frac{3\left(\alpha^{2}-1\right)}{4 \pi a^{3} \alpha^{3} N^{3 / 2}}+\frac{W_{1}}{2} \rho^{2}+W_{2} \rho^{3} \\
& -\frac{\pi f^{2}}{2\left(T^{*}\right)^{2}} \frac{a^{3}}{z_{0}\left(z_{0}+1\right)^{2}\left(1-B \rho_{0}\right)^{2}} \\
& \times\left[\frac{1}{\left(1-B \rho_{0}\right)^{2}}-\frac{1}{4 T^{*}} \frac{z_{0}}{\left(z_{0}+1\right)^{2}}\right]^{-1} \rho^{2}=0 .
\end{aligned}
$$

Note that this expression is just the minimization of the free energy of a usual polymer with the second virial coefficient renormalized to

$$
\begin{aligned}
\widetilde{W}_{1} \equiv & \frac{W_{1}}{2}-\frac{\pi f^{2}}{2\left(T^{*}\right)^{2}} \frac{a^{3}}{z_{0}\left(z_{0}+1\right)^{2}\left(1-B \rho_{0}\right)^{2}} \\
& \times\left[\frac{1}{\left(1-B \rho_{0}\right)^{2}}-\frac{1}{4 T^{*}} \frac{z_{0}}{\left(z_{0}+1\right)^{2}}\right]^{-1} .
\end{aligned}
$$

As in conventional polymers, for $\widetilde{W}_{1}>0(\operatorname{good}$ solvent case), the polymer will be fully extended, while for $\widetilde{W}_{1}<0$ (bad solvent case) will assume a globular form. At $T^{*}=T_{\theta}^{*}$ given by $\widetilde{W}_{1}=0$ in equation above ( $\theta$ solvent), there is a collapse transition, as shown in Fig. 2 and Fig. 3(b).

\section{APPENDIX C: THE FIRST-ORDER PHASE TRANSITION AT LOW TEMPERATURES WHEN $\rho_{0}<\rho_{c}$}

If the density of salt is chosen to be on the vapor side of the coexistence curve, at very low temperatures Eqs. (22), (23), and (24) exhibit two possible solutions: one with $\rho_{s}=\rho^{v}$ (low density of salt) and another with $\rho_{s}=\rho^{l}$ (high density of salt) as $T^{*} \rightarrow 0$. The first solution exists only for low temperatures, while the second is present for all temperatures. Consequently, we have to investigate if there would be a phase transition between these two phases as the temperature is decreased. In order to address this question, we have to compare the respective free energies for the two branches.

The free energy associated with the solution with low density of salt is obtained by substituting

$$
\begin{gathered}
x \approx 1+O\left(e^{-1 / 2 T^{*}}\right), \\
\alpha \approx \bar{\alpha}+O\left(e^{-1 / 2 T^{*}}\right), \\
\rho_{s} \approx \rho^{v}+O\left(e^{-1 / 2 T^{*}}\right),
\end{gathered}
$$

into the Eq. (19) what leads to

$$
\beta \mathcal{G}^{E} \approx-\frac{N f}{2 T^{*}}-\frac{N f}{2 \sqrt{\pi \rho_{M}^{*} T^{*}}}+O\left(e^{-1 / 2 T^{*}}\right) .
$$

On the other hand, the free energy associated with the high density state is obtained by substituting

$$
\begin{gathered}
x \approx 1+O\left(\frac{e^{-3 / 4 \sqrt{\pi \rho_{M}^{*} T^{*}}-\ln T^{*} / 8 \pi \rho_{M}^{*}}}{\sqrt{T^{*}}}\right), \\
\alpha \approx \bar{\alpha}+O(1-x), \\
\rho_{s} \approx \rho^{v}+O(1-x),
\end{gathered}
$$

into Eq. (19). The resulting free energy is given by

$$
\beta \mathcal{G}^{C} \approx-\frac{N f x}{2 T^{*}}-\frac{N f}{2 \sqrt{\pi \rho_{M}^{*} T^{*}}}+O\left(\frac{e^{-3 / 4} \sqrt{\pi \rho_{M}^{*} T^{*}}-\ln T^{*} / 8 \pi \rho_{M}^{*}}{\sqrt{T^{*}}}\right) .
$$

Comparing Eqs. (C2) and (C4) gives that $\beta \mathcal{G}^{C}$ 
$\approx \beta \mathcal{G}^{E}+(1-x) f N / 2 T^{*}, \quad$ where $(1-x)$ scales as $\exp \left\{-3 / 4 \sqrt{\pi \rho_{M}^{*} T^{*}}-\ln T^{*} / 8 \pi \rho_{M}^{*}\right\} / \sqrt{T^{*}}$. Since the last term in the above expression is positive for low temperatures, the phase of low density of salt is more stable at low tempera- tures. This indicates that there is a phase transition between the dilute globular state and the extended state. Unfortunately, numerical analysis indicates that this transition happens at very low temperature.
[1] P. G. de Gennes, J. Phys. Lett. 36, L-55 (1975).

[2] P. J. Flory, Principles of Polymer Chemistry (Cornell University Press, Ithaca, New York, 1953).

[3] P. G. de Gennes, Scaling Concepts in Polymer Physics (Cornell University Press, Ithaca, New York, 1979).

[4] P. G. de Gennes, Phys. Lett. 38A, 339 (1972).

[5] (a) G. S. Manning, J. Chem. Phys. 51, 924 (1969); (b) G. S. Manning, Q. Rev. Biophys. 11, 179 (1978); (c) Y. Levin, Europhys. Lett. 34, 405 (1996).

[6] M. Fixman, J. Chem. Phys. 70, 4995 (1979).

[7] T. Odjik, Macromolecules 12, 688 (1979).

[8] S. F. Edwards, P. R. King, and P. Pincus, Ferroelectrics 30, 7 (1980).

[9] P. G. Higgs and J. F. Joanny, J. Chem. Phys. 94, 1543 (1991).

[10] Y. Levin and M. C. Barbosa, Europhys. Lett. 31, 513 (1995).

[11] M. C. Barbosa and Y. Levin, Physica A 231, 467 (1996).

[12] P. W. Debye and E. Hückel, Phys. Z. 24, 185 (1923), for a good exposition, see D. A. MacQuarrie, Statistical Mechanics (Harper \& Row, New York, 1976), Chap. 15.

[13] N. Bjerrum, Kgs. Dan. Vidensk. Selsk. Mat.fys. Medd. 7, 1 (1926).

[14] P. J. Flory, Proc. R. Soc. London 351, 351 (1976).

[15] F. Tanaka and H. Ushiki, J. Chem. Phys. 84, 5925 (1986).

[16] A. Matsuyama and F. Tanaka, J. Chem. Phys. 94, 781 (1991).

[17] G. Allen, Proc. R. Soc. Lond. A 351, 381 (1976).
[18] P. G. de Gennes, P. Pincus, R. M. Velasco, and F. Brochard, J. Phys. (Paris) 1, 1461 (1976).

[19] For a review, see Y. Levin and M. E. Fisher, Physica A 225, 164 (1996).

[20] (a) M. E. Fisher and Y. Levin, Phys. Rev. Lett. 71, 3826 (1993); (b) M. E. Fisher, J. Stat. Phys. 75, 1 (1994).

[21] (a) Y. Levin, X.-J. Li, and M. E. Fisher, Phys. Rev. Lett. 73, 2716 (1994); (b) M. E. Fisher, X.-J. Li, and Y. Levin, J. Stat. Phys. 79, 1 (1995).

[22] Rajiv R. Singh and K. S. Pitzer, J. Chem. Phys. 92, 6775 (1992); T. Narayanan and K. S. Pitzer, Phys. Rev. Lett. 73, 3002 (1994).

[23] A. Z. Panangiotopoulos, Fluid Phase Equil. 76, 97 (1992).

[24] W. Ebeling, Z. Phys. Chem. 238, 400 (1968).

[25] For $T^{*}>0.2$ we have used the modified equilibrium constant derived by Ebeling

$$
\begin{aligned}
K^{E b}\left(T^{*}\right)= & \frac{2 \pi a^{3}}{3\left(T^{*}\right)^{3}}\left[\operatorname{Ei}\left(\frac{1}{T^{*}}\right)-\operatorname{Ei}\left(-\frac{1}{T^{*}}\right)+6 T^{*}+4\left(T^{*}\right)^{3}\right] \\
& -\frac{2 \pi a^{3}}{3} e^{1 / T^{*}}\left[2+\frac{1}{T^{*}}+\frac{1}{\left(T^{*}\right)^{2}}\right]-\frac{2 \pi a^{3}}{3} e^{-1 / T^{*}} \\
& \times\left[2-\frac{1}{T^{*}}+\frac{1}{\left(T^{*}\right)^{2}}\right] .
\end{aligned}
$$

[26] J. M. Corpart and F. Candau, Macromolecule 26, 1333 (1993). 